04

\title{
Особенности работы тиратрона с холодным катодом при наличии обратной полуволны напряжения
}

\author{
( Ю.Д. Королев, ${ }^{1,2,3}$ Н.В. Ландль, ${ }^{1,2}$ В.Г. Гейман, ${ }^{1}$ О.Б. Франц, ${ }^{1,2}$ А.В. Болотов, ${ }^{1}$ \\ B.O. Нехорошев, ${ }^{1,2}$ B.C. Касьянов ${ }^{1,2}$ \\ ${ }^{1}$ Институт сильноточной электроники СО РАН, \\ 634055 Томск, Россия \\ ${ }^{2}$ Томский государственный университет, \\ 634055 Томск, Россия \\ ${ }^{3}$ Томский политехнический университет, \\ 634055 Томск, Россия \\ e-mail: korolev@Inp.hcei.tsc.ru
}

\section{(Поступило в Редакцию 19 сентября 2016 г.)}

Исследованы особенности работы металлокерамического тиратрона типа TPI1-10k/50 в электрических цепях, включающих емкость, индуктивность и активное сопротивление при параметрах цепи, когда ток имеет колебательный характер. Эксперименты проведены при анодном напряжении до $30 \mathrm{kV}$, токе в прямом направлении до $7.6 \mathrm{kA}$ и длительности первого полупериода тока от $0.38 \mu \mathrm{s}$ до $1.9 \mu \mathrm{s}$. Получены данные по режимам работы, в которых тиратрон может коммутировать обратную волну тока и когда имеет место обрыв тока во втором полупериоде. Показано, что в процессе обрыва через тиратрон в обратном направлении протекает некоторый ток. Его величина и максимальное обратное напряжение на тиратроне определяют, будет ли происходить обрыв тока или повторный пробой прибора на обратном напряжении. При максимальном обратном токе на уровне нескольких сотен ампер полный обрыв тока происходит при обратных напряжениях вплоть до $12 \mathrm{kV}$. Обсуждены физические механизмы обрыва тока.

DOI: 10.21883/JTF.2017.05.44440.2039

\section{Введение}

В настоящее время довольно широкое применение получили коммутирующие приборы на основе сильноточных импульсных газовых разрядов низкого давления с полым катодом (так называемые псевдоискровые разрядники) [1-9]. Конструкция разрядника и принцип работы в значительной степени напоминают конструкцию классического водородного тиратрона с накаленным катодом. Однако в данном типе приборов накаленный катод отсутствует. Поэтому псевдоискровые разрядники часто называют тиратронами с холодным катодом, либо тиратронами с заземленной сеткой $[4,5,9]$.

На начальных стадиях коммутации разряд в тиратроне горит в форме импульсного тлеющего разряда с полым катодом, а на поздних стадиях на поверхности катода возникает катодное пятно и происходит переход тлеющего разряда в дуговой [4,5,10-17]. Часто в качестве достоинства тиратронов с холодным катодом указывается на возможность коммутирования колебательного тока [14], т.е. на возможность пропускания тока в обратном направлении, когда к аноду прибора прикладывается отрицательное напряжение. Однако такой режим наиболее просто получить лишь применительно к конструкциям, в которых анод является полым $[1,4,5,10,18]$. Если же анод плоский, то для поддержания тока в обратном направлении необходимо возникновение дугового анодного пятна с высокой эмиссионной способностью [18].

Тиратроны используются в самых различных электрических схемах, причем в некоторых схемах коммути- рование отрицательной волны напряжения нежелательно $[4,19]$. Здесь, напротив, необходимо иметь условия, когда к аноду прикладывается высокое отрицательное напряжение, причем прибор должен выдерживать это напряжение без пробоя. Соответственно проблема исследования работы приборов в электрических цепях с колебательным характером тока представляет интерес. В настоящей работе эта проблема исследуется применительно к отпаянному двухсекционному тиратрону типа TPI1-10k/50 [4,5].

\section{Экспериментальная установка и методика измерений}

Схема экспериментальной установки для исследования тиратрона показана на рис. 1. Тиратрон представляет собой отпаянный металлокерамический прибор. Рабочее давление газа в приборе поддерживается за счет генератора водорода, на который подается напряжение $V_{\mathrm{H}}$ на уровне $5-6 \mathrm{~V}$ при токе около $2 \mathrm{~A}[4,5]$. Условия горения разряда соответствуют левой ветви кривой Пашена, причем давление газа настолько низкое, что длина свободного пробега электрона для реакции ионизации превышает длину основного межэлектродного промежутка [4,20-25]. В таких условиях в отличие от разрядников высокого давления пробой в промежутке не может инициироваться одиночными электронами. Для инициирования пробоя необходим значительный поток электронов в промежуток со стороны катода $[4,10,25]$. 


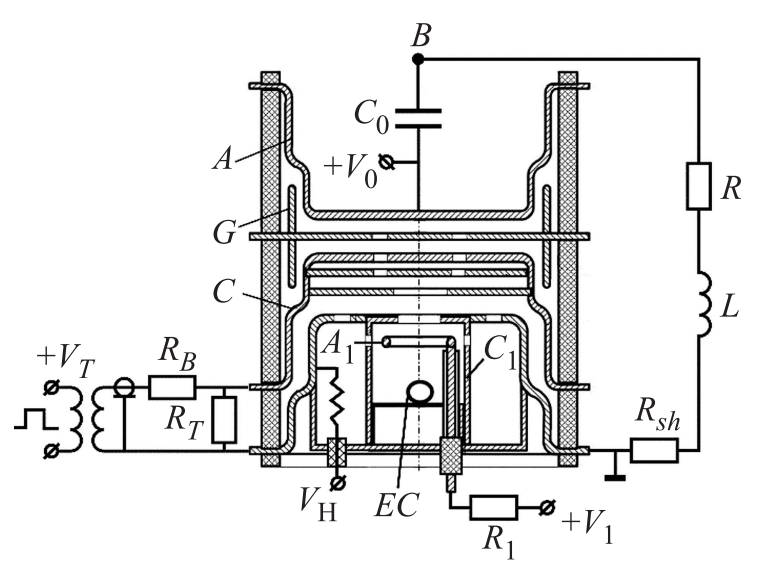

Pис. 1. Схематическое изображение тиратрона TPI1-10k/50 и электрическая цепь для исследования работы тиратрона. $A-$ анод тиратрона, $C$ - полый катод тиратрона, $G-$ промежуточный градиентный электрод, $A_{1}-$ кольцевой анод вспомогательного тлеющего разряда, $C_{1}$ - полый катод вспомогательного тлеющего разряда, $E C$ - таблетка с высокой эмиссионной способностью, $V_{0}$ - зарядное напряжение для накопительной емкости $C_{0}, V_{1}$ - напряжение источника для питания вспомогательного тлеющего разряда, $V_{T}$ - импульс для запуска тиратрона.

Этот поток обеспечивается за счет специального узла запуска разрядника. В исследуемом приборе узел запуска основан на вспомогательном тлеющем разряде $[4,5,26]$.

Основными электродами тиратрона, к которым прикладывается высокое напряжение $V_{0}$, являются плоский анод $A$ и полый катод $C$. Для повышения пробивного напряжения между основными электродами помещен градиентный электрод $G$. Этот электрод также защищает внутреннюю поверхность керамического корпуса от паров материала электродов. В полости основного катода $C$ располагается электродная система узла запуска, включающая в себя полый катод узла запуска $C_{1}$ и анод $A_{1}$ в форме кольца. Для экранировки основного межэлектродного промежутка от узла запуска полость катода $C$ имеет две перегородки с отверстиями.

Представленный на рис. 1 вариант включения прибора соответствует так называемой схеме классического тиратрона $[4,5]$. Здесь электрод $C_{1}$ заземлен, и электрод $C$ соединен с электродом $C_{1}$ через сопротивление $R_{T}=(0.5-1) \mathrm{k} \Omega$. Тогда в исходном состоянии электроды $C$ и $C_{1}$ находятся под нулевым потенциалом, и зарядное напряжение емкости $C_{0}$ приложено к аноду $A$.

Перед запуском прибора в электродной системе узла запуска между электродами $A_{1}$ и $C_{1}$ под действием напряжения $V_{1}$ зажигается слаботочный стационарный тлеющий разряд. Напряжение зажигания разряда (пробивное напряжение в системе электродов $A_{1} C_{1}$ ) менее $1 \mathrm{kV}$. Это напряжение, а также напряжения горения разряда и вольтамперная характеристика в значительной степени определяются свойствами так называемой таблетки с высокой эмиссионной способностью $E C[5,26]$. В рабочих режимах ток тлеющего разряда в узле запуска ограничивается балластным сопротивлением $R_{1}=(20-60) \mathrm{k} \Omega$ и обычно составляет 10-20 mA.

В представленной схеме тиратрон работает следующим образом. В исходном состоянии к аноду приложено напряжение $V_{0}$, и в узле запуска поддерживается слаботочный тлеющий разряд с полым катодом. Основной ток разряда замыкается на полость $C_{1}$. Тем не менее некоторая малая доля тока течет также через отверстие в электроде $C_{1}$ на электрод $C$. Для запуска тиратрона к электроду $C$ прикладывается импульс запуска положительной полярности $V_{T}=(2.5-5) \mathrm{kV}$. В результате в системе полых электродов $C$ и $C_{1}$ возникает импульсный разряд, ток которого ограничен балластным сопротивлением $R_{B}=120 \Omega$. Для разряда в цепи запуска электрод $C$ представляет собой полый анод сложной формы. Плазма разряда заполняет всю анодную полость, в том числе и пространство между верхней плоскостью электрода $C$ и средней перегородкой. Тогда электронный поток из этой плазмы через отверстия в верхней плоскости электрода $C$ будет извлекаться в основной промежуток. По сути, данная система представляет собой электронную пушку с плазменным катодом [4,5,22,27]. Благодаря потоку электронов в основной промежуток происходит формирование плазмы в электродной системе $A C$ по механизму, описанному в $[4,5,10,25]$, и инициирование сильноточного разряда.

Поскольку электроды $C$ и $C_{1}$ соединены между собой через сопротивление $R_{T}$, то ток сильноточного разряда уже в начальной стадии протекает с анода $A$ на полый катод $C_{1}$. В этом разряде эмиссия электронов обеспечивается главным образом потоком электронов из плазмы, которая формируется в полости катода $C_{1}$. Важную роль в поддержании плазмы в полом катоде $C_{1}$ играет таблетка с высокой эмиссионной способностью $E C[5,10,26]$. На следующей стадии на кромке выходного отверстия в электроде $C_{1}$ может возникать дуговое катодное пятно, которое является дополнительным источником эмиссии. В целом высокая эмиссионная способность катода $C_{1}$ является основным фактором, обеспечивающим поддержание сильноточного разряда в приборе при низком напряжении горения.

Как видно из рис. 1, при срабатывании тиратрона емкость $C_{0}$ разряжается через сопротивление $R$ и индуктивность $L$. В зависимости от соотношения между волновым сопротивлением электрической цепи и активным сопротивлением $R$ возможен апериодический либо колебательный характер тока. При колебательном токе во втором полупериоде к аноду прикладывается отрицательное напряжение, т. е. плоский электрод $A$ становится катодом. В общем случае, если катод не обеспечивает достаточную эмиссию электронов, то может наблюдаться резкое увеличение сопротивления прибора и уменьшение тока во втором полупериоде, вплоть до полного обрыва тока. Исследование особенностей протекания тока в тиратроне во втором полупериоде и является основным направлением представленной работы. 


\section{Схематическое изображение осциллограмм тока и напряжения в различных режимах коммутации}

На рис. 2, а представлена в более наглядном виде электрическая схема для исследования тиратрона. На ней прибор изображен в виде идеального ключа $S$, который коммутирует на землю емкость $C_{0}$, заряженную в исходном состоянии до напряжения $V_{0}$. Отдельно показано сопротивление разрядника $R_{S}$. Оно в общем случае изменяется во времени. В цепи имеются активное сопротивление $R$ и индуктивность $L$. Сигнал тока на осциллограф снимается с низкоомного шунта $R_{s h}=0.03 \Omega$. Изменение напряжения во времени регистрируется между точкой $B$ и землей, т.е. измеряется падение напряжения $V_{B}=V_{R}+V_{L}$. Соответственно полное активное сопротивление электрической цепи, определяющее характер затухания тока, $R_{t o t}=R+R_{S}$.

На рис. 2, $b$ представлены расчетные осциллограммы тока и напряжения для случая, когда тиратрон пропускает положительную и отрицательную полуволны тока. Чтобы условия протекания тока были близки к экспериментальным, в расчетах мы взяли следующие параметры электрической цепи: $C_{0}=47 \mathrm{nF}, L=0.85 \mu \mathrm{H}$, $R=0.2 \Omega, R_{S}=0.38 \Omega$. Ток нормирован на величину $i_{0}=V_{0} / \rho$, где $\rho=\left(L / C_{0}\right)^{1 / 2}-$ волновое сопротивление электрической цепи.

В данном случае мы имеем ситуацию, когда активное сопротивление колебательного контура $R_{t o t}=0.58 \Omega$ намного меньше волнового сопротивления $\rho=4.3 \Omega$. Тогда при срабатывании разрядника напряжение на точке $B$ становится отрицательным и приблизительно равным $V_{0}$. Ток имеет колебательный характер с затуханием, определяемом сопротивлением $R_{t o t}$. Время $t_{4}$ практически соответствует периоду свободных колебаний в контуре $T=2 \pi\left(L C_{0}\right)^{1 / 2}$.

Идеализированные осциллограммы, которые мы могли бы получать в случае, когда сопротивление разрядника $R_{S}$ резко возрастает в момент перехода тока

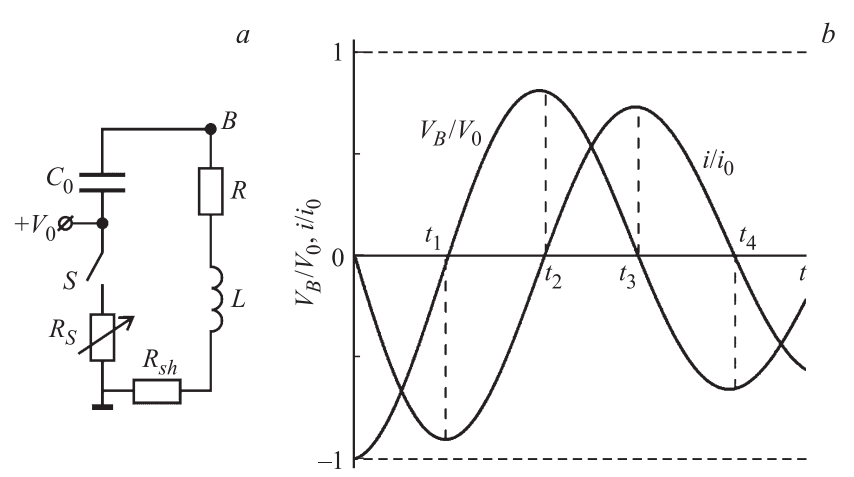

Рис. 2. Электрическая схема измерений $(a)$ и схематическое изображение осциллограммы тока в цепи и напряжения на точке $B$ относительно земли $(b)$ для условий, когда тиратрон пропускает обратную полуволну тока и обрыв тока во втором полупериоде отсутствует. через ноль (в момент начала второго полупериода $t_{2}$ ), представлены на рис. 3. Если сопротивление разрядника скачком становится равным бесконечности, то происходит полный обрыв тока в цепи (рис. 3,a). Это значит, что напряжение на точке $B$ становится равным нулю, и к аноду разрядника прикладывается напряжение отрицательной полярности $V_{B}\left(t_{2}\right)$.

Другой схематический случай показан на рис. $3, b$. Здесь в момент $t_{2}$ сопротивление разрядника скачком увеличивается до величины $R_{S}\left(t_{2}\right)=3 \rho$. Соответственно для времени $t \geq t_{2}$ имеет место апериодический разряд конденсатора через активное сопротивление $R_{t o t}$. Характерное время разрядки конденсатора соответствует $R_{t o t} C_{0}$. В некоторый момент времени $t_{m}$ ток достигает максимального значения. Поскольку активное сопротивление в цепи определяется главным образом сопротивлением разрядника и индуктивное падение напряжения равно нулю, напряжение $V_{B}\left(t_{m}\right)$ оказывается близким к нулю. Тогда для $t>t_{m}$ отрицательное напряжение $V_{B}$ представляет собой падение напряжения на индуктивности при спаде тока.

\section{Результаты и обсуждение}

Как отмечалось, экспериментальные результаты были получены на отпаянном двухсекционном тиратроне типа TPI1-10k/50. Анод прибора был плоским. Давление газа в приборе определяется напряжением на генераторе водорода, которое в экспериментах изменялось в пределах $V_{\mathrm{H}}=(5.8-6.2) \mathrm{V}$. Соответственно при $V_{\mathrm{H}}=5.8 \mathrm{~V}$ статическое пробивное напряжение основного межэлектродного промежутка $A C$ было максимальным и составляло около $30 \mathrm{kV}$, а при напряжении $V_{\mathrm{H}}=6.2 \mathrm{~V}$ оно снижалось примерно до $8 \mathrm{kV}$. Использовались электрические цепи с емкостями 47, 94 и $188 \mathrm{nF}$. Индуктивность могла меняться от 0.32 до $2 \mu \mathrm{H}$. Пример осциллограмм тока и напряжения для условий, когда имеет место полный обрыв тока во втором полупериоде, показан на рис. 4.

В первом полупериоде тока активное сопротивление электрической цепи $R_{t o t}$ намного меньше волнового сопротивления. Соответственно длительность $t_{2}$ оказывается близкой к половине периода свободных колебаний $T / 2$. Тогда, обрабатывая осциллограмму тока, мы можем определить длительность первого полупериода $t_{2} \approx T / 2=630 \mathrm{~ns}$. Отсюда получаем индуктивность электрической цепи $L=0.85 \mu \mathrm{H}$ и волновое сопротивление $\rho=4.3 \Omega$.

При начальном напряжении на емкости $V_{0}=16 \mathrm{kV}$ запасенная в ней энергия $W_{0}$ составляет $6.02 \mathrm{~J}$. Энергию, оставшуюся в электрической цепи к моменту времени $t_{1}$, можем оценить по току $i\left(t_{1}\right)=3235 \mathrm{~A}$ и известной индуктивности как $W\left(t_{1}\right)=4.45 \mathrm{~J}$. В момент времени $t_{2}$ емкость полностью перезаряжается. Напряжение на емкости составляет $V_{C}\left(t_{2}\right)=12.9 \mathrm{kV}$ и запасенная энергия $W\left(t_{2}\right)=3.91 \mathrm{~J}$. 

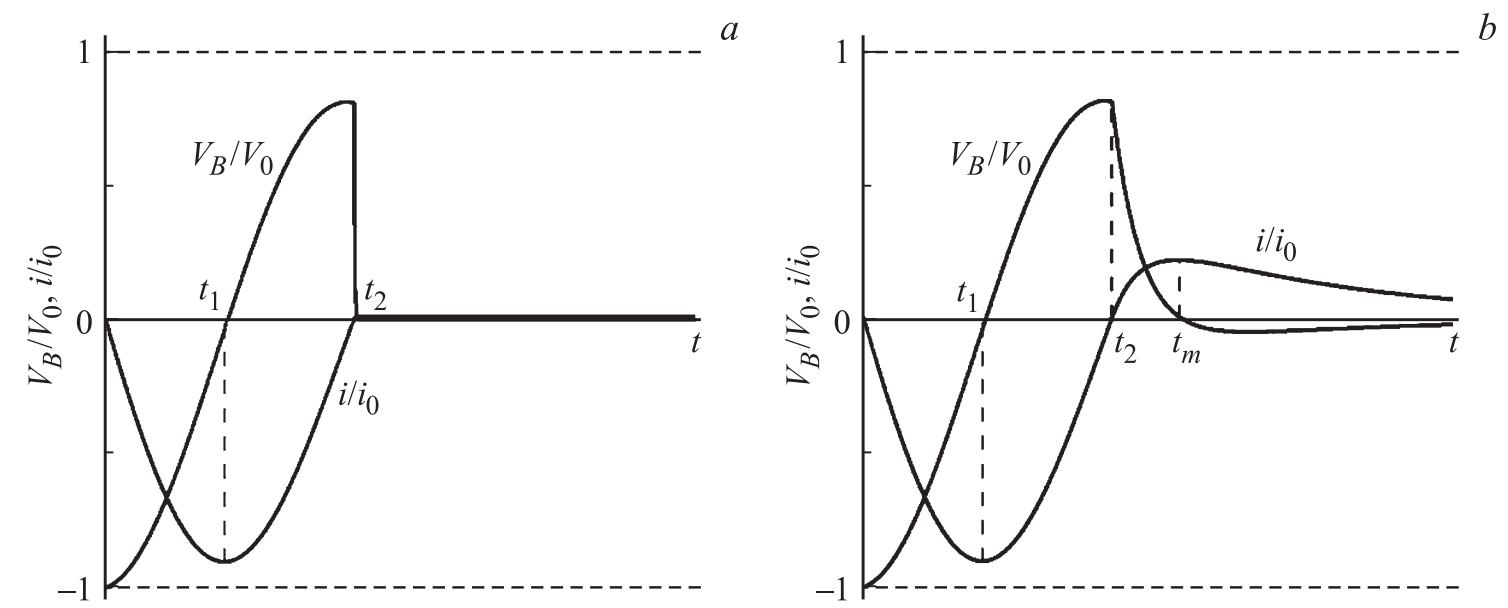

Рис. 3. Схематическое изображение осциллограмм в условиях резкого увеличения сопротивления разрядника в момент $t_{2}$. $a-$ в момент $t_{2}$ происходит обрыв тока, т. е. сопротивление разрядника скачком становится равным бесконечности. $b-$ в момент $t_{2}$ сопротивление разрядника становится равным $3 \rho$.

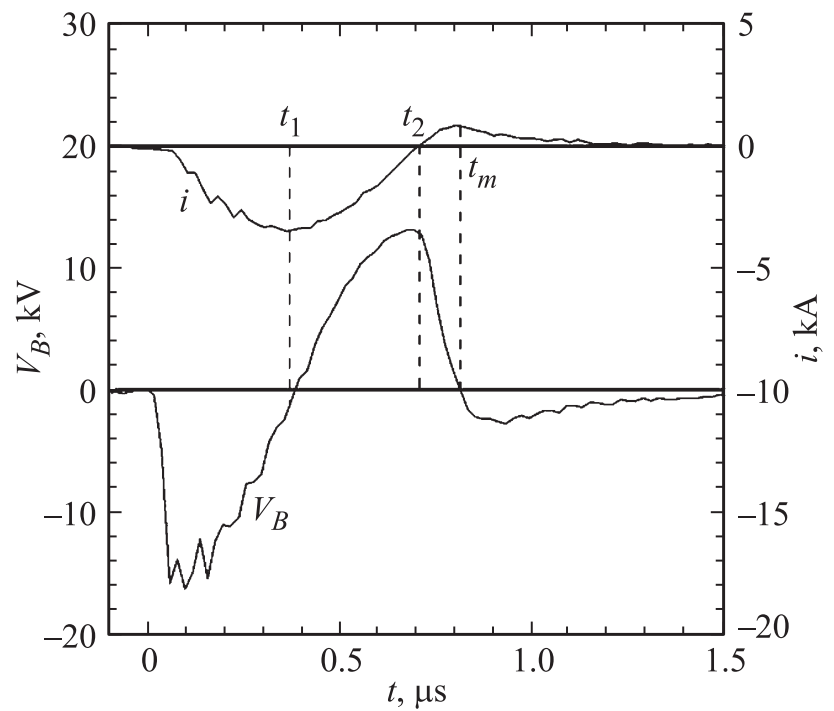

Рис. 4. Осциллограммы тока и напряжения в условиях полного обрыва тока во втором полупериоде. $V_{0}=16 \mathrm{kV}, V_{\mathrm{H}}=6 \mathrm{~V}$, $C_{0}=47 \mathrm{nF}, L=0.85 \mu \mathrm{H}, R=0.2 \Omega, \rho=4.3 \Omega, T / 2=630 \mathrm{~ns}$.

Затухание тока в течение первого полупериода определяется активным сопротивлением электрической цепи, включающем в себя сопротивление $R$ и некоторое усредненное сопротивление разрядника $R_{S}$. Принимая, что коэффициент затухания $\beta=R_{t o t} / 2 L$, можем определить $R_{t o t}=0.58 \Omega$. Тогда сопротивление разрядника $R_{S}=0.38 \Omega$, а падение напряжения на разряднике $V_{S}\left(t_{1}\right)=1.4 \mathrm{kV}$. С учетом того, что разрядник двухсекционный и режим протекания тока соответствует сверхплотному тлеющему разряду, оцененное падение напряжения представляется вполне разумным $[4,10]$.

Таким образом, к началу второго полупериода тока имеем следующую ситуацию. Емкость $C_{0}$, заряженная до напряжения $V_{C}\left(t_{2}\right)=12.9 \mathrm{kV}$, начинает разряжаться по цепи $L R_{t o t}$. Если бы сопротивление $R_{S}$ во втором полупериоде после момента $t_{2}$ не изменилось, то мы бы регистрировали осциллограмму, сходную со схематически показанной на рис. $2, b$. Если бы произошло мгновенное увеличение сопротивления разрядника до бесконечности, то ток в цепи стал бы равным нулю, и мы бы регистрировали осциллограмму, показанную на рис. 3, $a$. Однако мы видим, что на начальной стадии сопротивление $R_{S}$ возрастает, но не до бесконечности, и экспериментальная осциллограмма по виду напоминает расчетную осциллограмму, приведенную на рис. $3, b$.

В соответствии со схемой, представленной на рис. $2, a$, мы измеряем падение напряжения на последовательно соединенных сопротивлении $R$ и индуктивности $L$. Оно не будет равно напряжению на емкости, поскольку часть напряжения $V_{C}$ выпадет на сопротивлении разрядника $R_{S}$. На осциллограмме тока после момента $t_{2}$ наблюдается максимум с током $i\left(t_{m}\right)=880 \mathrm{~A}$. Тогда падение напряжения на сопротивлении $R$ в момент $t_{m}$ определяется как $V_{B}\left(t_{m}\right)=i\left(t_{m}\right) R=176 \mathrm{~V}$. В масштабе шкалы осциллограммы это напряжение практически равно нулю. С учетом заряда, который стекает с емкости в интервале времени от $t_{2}$ до $t_{m}$, легко оценить напряжение на емкости $V_{C}\left(t_{m}\right)=11860 \mathrm{~V}$. Именно данное напряжение и приложено к разряднику в момент $t_{m}$. Соответственно сопротивление разрядника в момент $t_{m}$ оказалось $R_{S}\left(t_{m}\right)=13.5 \Omega$. В дальнейшем сопротивление разрядника продолжает нарастать, что обусловливает спад тока в цепи вплоть до полного прекращения тока, т.е. до обрыва тока. Отрицательное падение напряжения $V_{B}(t)$ после момента $t_{m}$ представляет собой индуктивное падение, обусловленное уменьшением тока во времени. Остаточное напряжение на емкости после обрыва тока оказалось равным $6.5 \mathrm{kV}$.

Таким образом, для рассматриваемого примера можно выделить некоторые важные особенности. Во второй полуволне тока происходит быстрое увеличение сопро- 

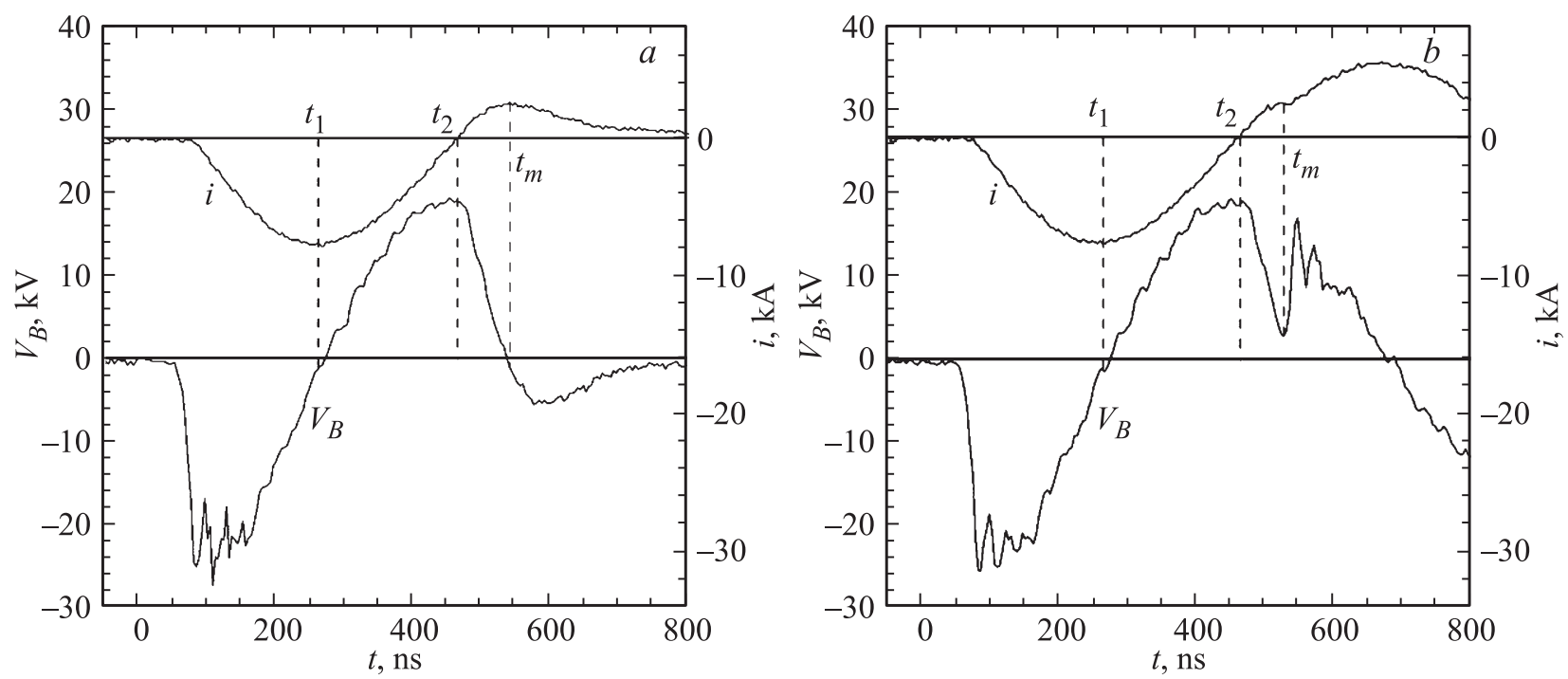

Рис. 5. Осциллограммы тока и напряжения в условиях полного $(a)$ обрыва тока во втором полупериоде и неполного обрыва $(b)$. $V_{0}=23 \mathrm{kV}, V_{\mathrm{H}}=5.8 \mathrm{~V}, C_{0}=47 \mathrm{nF}, L=0.32 \mu \mathrm{H}, R=0.27 \Omega, \rho=2.61 \Omega, T / 2=385 \mathrm{~ns}$.

тивления разрядника $R_{S}$. Оно за характерное время около $100 \mathrm{~ns}$ становится существенно больше волнового сопротивления электрической цепи. Хотя в начальной стадии тиратрон пропускает некоторый обратный ток, но приложенное к аноду отрицательное напряжение $V_{S}\left(t_{m}\right)$ на уровне $12 \mathrm{kV}$ не приводит к обратному пробою в тиратроне. Полный обрыв тока с сохранением остаточного напряжения на емкости происходит за время около $500 \mathrm{~ns}$.

Процессы в тиратроне после момента времени $t_{2}$ на качественном уровне можно интерпретировать следующим образом. Полый катод $C_{1}$ становится полым анодом, а электрод $A$ становится катодом. Тогда из плазмы полости $C_{1}$ и из плазмы полостей электрода $C$ в основной межэлектродный промежуток извлекается поток ионов. По своему устройству система напоминает собой источник ионов с плазменным катодом $[18,28,29]$. Ток в такой системе ограничивается межэлектродным промежутком $A G$, в котором ток переносится как ионами, так и электронами.

Электроны плазмы, которая была сформирована в промежутке $A G$, уходят от электрода $A$, и вблизи него образуется некомпенсированный объемный заряд положительных ионов, т.е. вблизи плоского электрода формируется область прикатодного падения потенциала тлеющего разряда $[10,18,30-32]$. Сопротивление промежутка определяется данной областью, и в случае плоского электрода оно оказывается довольно высоким. Действительно, основной ток в прикатодной области переносится ионами, а электроны возникают на катоде лишь за счет $\gamma$-процессов. Чтобы промежуток переносил большой ток при малом падении напряжения на нем, необходимо иметь высокий ток эмиссии с катода.

В работе [18] показано, что если бы электрод $A$ был полым, то ситуация была бы существенно другой. В этом случае во втором полупериоде тока в промежутке возникает сильноточный импульсный тлеющий разряд с полым катодом, и прибор оказывается способным коммутировать ток в обратном направлении. Именно поэтому в разрядниках, предназначенных для работы в цепях с колебательным характером тока, используются приборы с полым анодом [4].

Для рассматриваемого прибора с плоским анодом мы имеем другую ситуацию. В момент времени $t_{m}$ к разряднику приложено напряжение около $12 \mathrm{kV}$, причем все это напряжение сосредоточено на зазоре $A G$. Дальнейшее развитие разряда определяется тем, является ли напряженность поля на поверхности электрода $A$ достаточной для того, чтобы за счет взрывоэмиссионных процессов на этом электроде образовалось катодное пятно. При возникновении пятна ток эмиссии будет резко возрастать, сопротивление промежутка уменьшится, и фактически произойдет пробой промежутка на обратном напряжении.

Применительно к рассматриваемому примеру катодное пятно не возникает. При протекании обратного тока происходит уменьшение плотности плазмы в полостях $C_{1}$ и $C$. Это уменьшение связано главным образом с тем, что электроны и ионы уходят на электроды, причем уход заряженных частиц не компенсируется за счет процессов ионизации [30,32]. В конечном счете заряды полностью извлекаются из промежутка и ток прекращается. Здесь имеет место полный обрыв тока при остаточном напряжении на емкости на уровне $6 \mathrm{kV}$.

На рис. 5 представлены осциллограммы для режима, когда в одних и тех же условиях от импульса к импульсу может иметь место случай как полного обрыва тока и так называемого частичного (неполного) обрыва тока. По сравнению с условиями рис. 4 индуктивность цепи уменьшена до $L=0.32 \mu \mathrm{H}$, а начальное 

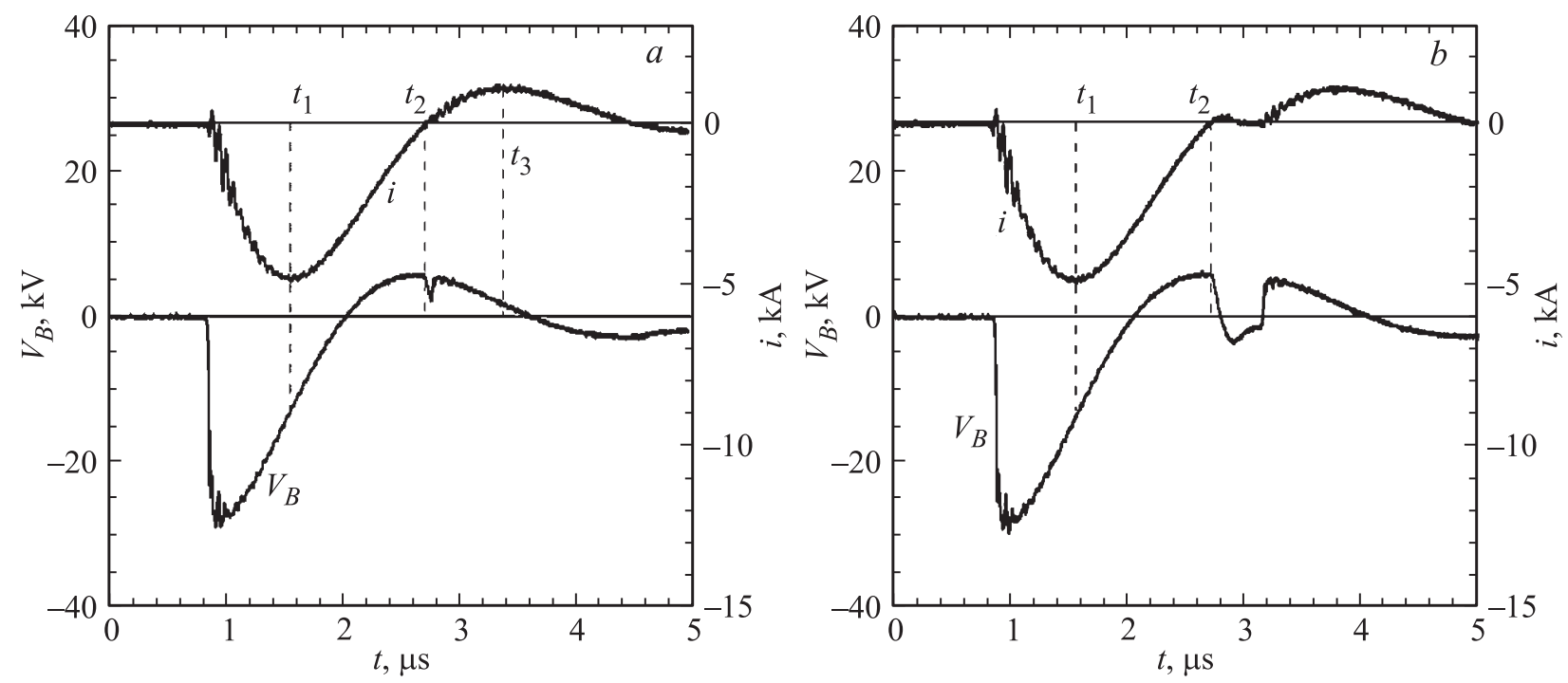

Рис. 6. Осциллограммы тока и напряжения в условиях отсутствия обрыва тока во втором полупериоде $(a)$ и неполного обрыва $(b)$. $V_{0}=30 \mathrm{kV}, V_{\mathrm{H}}=5.8 \mathrm{~V}, C_{0}=188 \mathrm{nF}, L=2 \mu \mathrm{H}, R=2.4 \Omega, \rho=3.26 \Omega, T / 2=1.92 \mu \mathrm{s}$.

напряжение на емкости увеличено до $V_{0}=23 \mathrm{kV}$. Соответственно ток в первом полупериоде был увеличен до $i\left(t_{1}\right)=7635 \mathrm{~A}$. Оценка усредненного сопротивления разрядника в течение первого полупериода тока дает величину $R_{S}=0.21 \Omega$. К началу второго полупериода емкость оказалась заряженной до напряжения $V_{C}\left(t_{2}\right)=17.25 \mathrm{kV}$.

Исходя из качественного описания механизма обрыва тока, можно заключить, что на процесс обрыва должно влиять характерное время нарастания тока до максимума во втором полупериоде, сама величина тока в момент $t_{m}$ и напряжение на емкости в момент $t_{m}$. Напомним, что последняя величина фактически представляет собой обратное напряжение, приложенное к разряднику, $V_{C}\left(t_{m}\right) \approx V_{S}\left(t_{m}\right)$.

Из сравнения рис. 4, 5 видно, что характерное время достижения максимума тока для обоих случаев оказалось примерно одинаковым и равным $100 \mathrm{~ns}$. Тем не менее в низкоиндуктивной электрической цепи величина заряда, стекающего с емкости за данное время, была больше. Поэтому обратные напряжения на разряднике в момент $t_{m}$ оказались соизмеримыми. Наибольшая разница имеет место в токе, достигаемом к моменту $t_{m}$. В частности, для условий рис. 5 этот ток составляет 2500 А, т. е. в 2.8 раза больше, чем для условий рис. 4.

По-видимому, высокая концентрация плазмы в зазоpe $A G$ при большом токе способствуют тому, что при формировании катодного падения потенциала напряженность поля на катоде становится достаточной для формирования катодного пятна. На некоторых осциллограммах (пример одной из них показан на рис. $5, b$ ) мы видим вначале стадию увеличения сопротивления промежутка, т. е. замедления роста тока и увеличения напряжения на разряднике. Эту стадию можно интерпретировать как начальную стадию обрыва тока. Тем не менее полного обрыва тока не происходит. В момент $t_{m}$ формируется катодное пятно, что приводит к уменьшению сопротивления разрядника и к дальнейшему колебательному характеру тока.

Таким образом, в общем случае особенности обрыва тока определяются характеристиками электрической цепи и условиями в разряднике к началу второго полупериода, т.е. моменту $t_{2}$. На первоначальной стадии развития процесса обрыва имеется характерное время (около $100 \mathrm{~ns}$ ), в течение которого обратный ток через разрядник достигает максимума. Именно в момент $t_{m}$ обратное напряжение, приложенное к разряднику, также является максимальным. Ясно, что снижение обратного тока и обратного напряжения способствует полному обрыву тока. В рассмотренных электрических цепях устойчивый полный обрыв тока наблюдался для токов $i\left(t_{m}\right)$ вплоть до $2 \mathrm{kA}$, причем обратное напряжение в процессе обрыва $V_{S}\left(t_{m}\right)$ могло составлять до $12 \mathrm{kV}$.

Проведенное рассмотрение относится к случаю, когда в течение первого полупериода разряд в тиратроне горит в форме импульсного тлеющего разряда с полым катодом и плоским анодом, причем анодное пятно отсутствует. Однако вполне можно представить себе ситуацию, в которой анодное пятно появляется уже в первом полупериоде тока. Это может быть, в частности, если переносимый в течение первого полупериода заряд достаточен для инициирования анодного пятна. Тогда при смене полярности тока анодное станет катодным пятном и обрыва тока вообще не будет.

Чтобы получить обсуждаемую ситуацию, мы увеличили емкость до величины $C_{0}=188 \mathrm{nF}$, а начальное напряжение на емкости также увеличили до $V_{0}=30 \mathrm{kV}$. Пример осциллограмм, полученных в данном режиме, 
представлен на рис. 6. Максимальный ток в первом полупериоде был $i\left(t_{1}\right)=4900 \mathrm{~A}$. Ток разряда имеет колебательный характер, хотя при используемом внешнем сопротивлении $R=2.4 \Omega$ коэффициент затухания тока возрос и сама форма тока в первом полупериоде несколько отклоняется от синусоидальной. Заряд, переносимый в первом полупериоде, был увеличен примерно в три раза по сравнению с условиями, представленными на рис. 5 , и составил около $6 \cdot 10^{-3} \mathrm{C}$.

Представляется, что для такой величины переносимого заряда уже возможен режим, когда в первом полупериоде на некоторых импульсах возникает анодное пятно. Осциллограммы для данного режима показаны на рис. 6,a. Здесь на токе не наблюдается никаких изменений, связанных с развитием процесса обрыва тока. Можно сказать, что обрыв тока фактически отсутствует, и осциллограммы по внешнему виду соответствуют представленным на рис. 2. В момент перехода тока через ноль на осциллограмме $V_{B}(t)$ имеется некоторый кратковременный спад напряжения. Он происходит за счет увеличения сопротивления разрядника при смене полярности напряжения на электродах, т.е. на временном интервале, когда вблизи электрода $A$ формируется катодный слой.

Обсуждаемый режим является критическим в том смысле, что анодное пятно в первом полупериоде образуется не на всех импульсах (переносимый заряд недостаточно велик, чтобы вероятность образования пятна была равна единице). Поэтому на некоторых импульсах во втором полупериоде имеют место неполные обрывы тока. Пример соответствующей осциллограммы показан на рис. $6, b$. После момента $t_{2}$ мы можем выделить стадию роста тока до величины $i\left(t_{m}\right)$ длительностью около $100 \mathrm{~ns}$. Тем не менее величина тока $i\left(t_{m}\right)$ мала (около $150 \mathrm{~A}$ ), поскольку в электрической цепи с большой индуктивностью ток не может достичь большой величины за короткое время. Напряжение на разряднике в момент $t_{m}$ составило $V_{S}\left(t_{m}\right) \approx V_{B}\left(t_{2}\right)=6 \mathrm{kV}$. Тем не менее, несмотря на малый ток и небольшое обратное напряжение на разряднике, полного обрыва тока не происходит. Спустя $400 \mathrm{~ns}$ после момента $t_{2}$ регистрируется повторный пробой промежутка на обратном напряжении.

Если снизить начальное напряжение, то заряд, переносимый в первом полупериоде, уменьшается. Типичные осциллограммы для напряжения $V_{0}=16 \mathrm{kV}$ показаны на рис. 7. Видно, что здесь имеет место случай полного обрыва тока. При этом вид экспериментальных осциллограмм близок к их схематическому изображению, представленному на рис. 3, $a$. Ясно, что увеличение сопротивления разрядника до бесконечности происходит не мгновенно. Соответственно на экспериментальной осциллограмме различим интервал от $t_{2}$ до $t_{m}$. Однако в электрической цепи с большой индуктивностью процесс выхода тока на значение $i\left(t_{m}\right)$ регистрируется не столь отчетливо, как, например, на рис. 4. Отметим, что для напряжения $V_{0}=16 \mathrm{kV}$ полный обрыв тока также

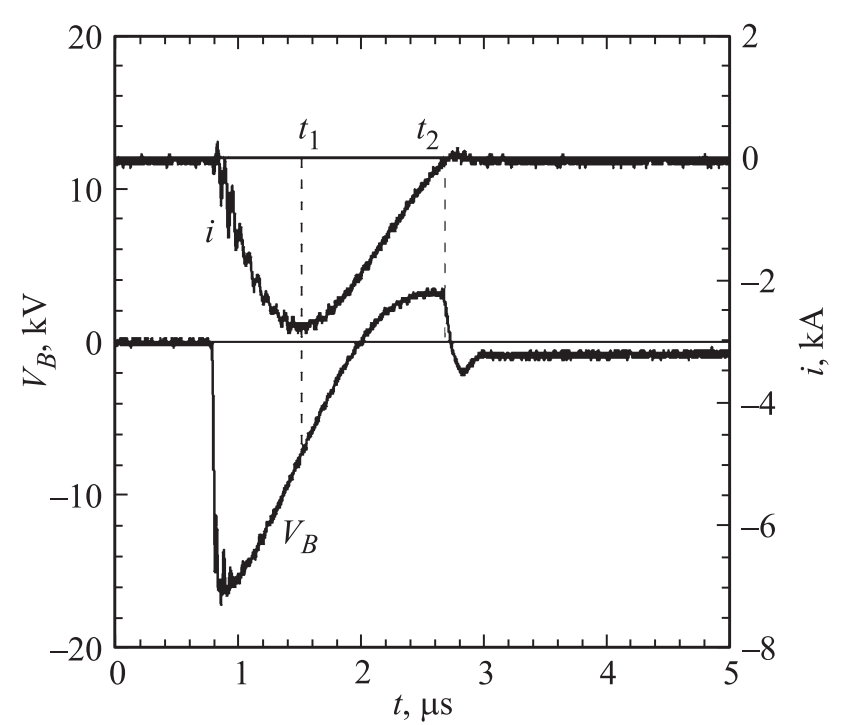

Рис. 7. Осциллограммы тока и напряжения в условиях полного обрыва тока во втором полупериоде. $V_{0}=16 \mathrm{kV}, V_{\mathrm{H}}=6 \mathrm{~V}$, $C_{0}=188 \mathrm{nF}, L=2 \mu \mathrm{H}, R=2.4 \Omega, \rho=3.26 \Omega, T / 2=1.92 \mu \mathrm{s}$.

происходит не во всех импульсах. Для того чтобы вероятность обрыва была близка к единице, напряжение $V_{0}$ необходимо снизить до $10 \mathrm{kV}$. Тогда обратное напряжение на разряднике в процессе обрыва составляет $V_{S}\left(t_{m}\right) \approx V_{B}\left(t_{2}\right)=2 \mathrm{kV}$.

\section{Заключение}

Исследованы особенности работы отпаянного металлокерамического тиратрона с холодным катодом типа TPI1-10k/50 в электрических цепях, включающих емкость, индуктивность и активное сопротивление, при параметрах цепи, когда ток имеет колебательный характер. Обсуждаются условия, в которых тиратрон может коммутировать обратную волну тока и когда имеет место обрыв тока во втором полупериоде.

Обрыв тока наблюдается для конструкций тиратрона с плоским анодом, причем необходимо, чтобы в первом полупериоде при протекании прямого тока на плоском аноде не возникало анодное пятно. Тогда при переходе тока через ноль анод прибора становится катодом, вблизи катода формируется область катодного падения потенциала импульсного тлеющего разряда, и сопротивление прибора в целом определяется данной областью.

Во втором полупериоде через тиратрон протекает некоторый ток, определяемый сопротивлением прибора, которое увеличивается во времени. Ток достигает максимума за характерное время около $100 \mathrm{~ns}$, а затем уменьшается вплоть до полного прекращения. В момент максимума тока обратное напряжение, приложенное к электродам, максимально. Это напряжение и максимальный обратный ток являются основными параметрами, определяющими, произойдет ли обрыв тока 
или будет иметь место повторный пробой прибора на обратном напряжении. При прямом токе через тиратрон на уровне $3 \mathrm{kA}$ и при максимальном обратном токе на уровне нескольких сотен ампер полный обрыв тока происходит при обратных напряжениях вплоть до $12 \mathrm{kV}$. Причиной скачкообразного уменьшения сопротивления прибора в процессе обрыва тока, т.е. пробоя на обратном напряжении, является образование катодного пятна.

Работа поддержана грантом Российского научного фонда, проект № 14-19-00139.

\section{Список литературы}

[1] Frank K., Christiansen J. // IEEE Trans. Plasma Sci. 1989. Vol. 17. N 5. P. 748-753.

[2] Bickel P., Christiansen J., Frank K., Gortler A., Hartmann W., Kowalewicz R., Linsenmeyer A., Kozlik C., Stark R., Wiesneth P. // IEEE Trans. Electron Devices. 1991. Vol. 38. N 4. P. $712-716$.

[3] Frank K., Dewald E., Bickes C., Ernst U., Iberler M., Meier J., Pruker U., Rainer A., Schlaug M., Schwab J. // IEEE Trans. Plasma Sci. 1999. Vol. 27. N 4. P. 1008-1020.

[4] Bochkov V.D., Dyagilev V.M., Ushich V.G., Frants O.B., Korolev Y.D., Shemyakin I.A., Frank K. // IEEE Trans. Plasma Sci. 2001. Vol. 29. N 5. P. 802-808.

[5] Korolev Y.D., Landl N.V., Geyman V.G., Bolotov A.V., Kasyanov V.S., Nekhoroshev V.O., Kovalsky S.S. // IEEE Trans. Plasma Sci. 2015. Vol. 43. N 8. P. 2349-2353.

[6] Lamba R.P., Pathania V., Meena B.L., Rahaman H., Pal U.N., Prakash R. // Rev. Sci. Instrum. 2015. Vol. 86. N 10. P. 103508

[7] Meena B.L., Rai S.K., Tyagi M.S., Pal U.N., Kumar M., Sharma A.K. // J. Phys. Conference Series. 2010. Vol. 208. P. 012110.

[8] Zhang J., Zhao J.P., Zhang Q.G. // IEEE Trans. Plasma Sci. 2014. Vol. 42. N 8. P. 2037-2041.

[9] Voitenko N.V., Yudin A.S., Kuznetsova N.S., Bochkov V.D. // J. Phys. Conference Series. 2015. Vol. 652. P. 012059.

[10] Korolev Y.D., Frants O.B., Landl N.V., Shemyakin I.A., Geyman V.G. // IEEE Trans. Plasma Sci. 2013. Vol. 41. N 8. P. 2087-2096.

[11] Kondrat'eva N.P., Koval N.N., Korolev Y.D., Schanin P.M. // J. Phys. D: Appl. Phys. 1999. Vol. 32. P. 699-705.

[12] Королев Ю.Д., Месяи Г.А., Хузеев А.П. // Докл. АН СССР. 1980. T. 253. № 3. С. 606-609.

[13] Козырев А.В., Королев Ю.Д., Месяи Г.А., Новоселов Ю.Н., Шемякин И.А. // ЖТФ. 1981. Т. 51. Вып. 9. С. 1817-1822.

[14] Korolev Y.D., Mesyats G.A., Yarosh A.M. // High Energy Chem. 1987. Vol. 21. N 5. P. 389-392.

[15] Hu J., Rovey J.L. // J. Appl. Phys. 2013. Vol. 114. N 7. P. 073301.

[16] Feng J.H., Zhou L., Fu Y.C., Zhang J.H., Xu R.K., Chen F.X., Li L.B., Meng S.J. // AIP Advances. 2014. Vol. 4. N 7. P. 077115.

[17] Benilov M.S., Benilova L.G. // IEEE Trans. Plasma Sci. 2015. Vol. 43. N 8. P. 2247-2252.

[18] Frank K., Korolev Y.D., Kuzmichev A.I. // IEEE Trans. Plasma Sci. 2002. Vol. 30. N 1. P. 357-362.
[19] Акимов А.В., Бак П.А., Корепанов А.А., Логачев П.В., Бочков В.Д., Бочков Д.В., Дягилев В.М., Ушич В.Г. // Вестник НГУ. Сер. Физика. 2008. Т. 3. Вып. 4. С. 68-73.

[20] Koval N.N., Ivanov Y.F., Lopatin I.V., Akhmadeev Y.H., Shugurov V.V., Krysina O.V., Denisov V.V. // Russ. J. Gen. Chem. 2015. Vol. 85. N 5. P. 1326-1338.

[21] Казаков А.В., Медовник А.В., Бурдовицин В.А., Окс Е.М. // ЖТФ. 2015. Т. 85. Вып. 2. С. 55-58.

[22] Гаврилов Н.В., Меньшаков А.И. // ЖТФ. 2016. Т. 86. Вып. 5. C. 30-36.

[23] Ни J., Rovey J.L. // J. Phys. D: Appl. Phys. 2012. Vol. 45. N 46. P. 465203.

[24] Devyatkov V.N., Koval N.N., Schanin P.M., Grigoryev V.P., Koval T.V. // Laser Part. Beams. 2003. Vol. 21. N 2. P. 243248.

[25] Kozyrev A.V., Korolev Y.D., Rabotkin V.G., Shemyakin I.A. // J. Appl. Phys. 1993. Vol. 74. N 9. P. 5366-5371.

[26] Королев Ю.Д., Ландль Н.В., Гейман В.Г., Франи О.Б., Шемякин И.А., Нехорошев В.О. // Физика плазмы. 2016. Т. 42. № 8. С. 775-784.

[27] Koval T., Devyatkov V.N., Hung N.V. // J. Phys. Conference Series. 2015. Vol. 652. P. 012061.

[28] Ryabchikov A.I., Ryabchikov I.A., Stepanov I.B., Usov U.P. // Surface Coatings Tech. 2007. Vol. 201. N 15. P. 6523-6525.

[29] Ryabchikov A.I. // Rev. Sci. Instruments. 1992. Vol. 63. N 4. P. 2425-2427.

[30] Korolev Y.D., Frants O.B., Geyman V.G., Kasyanov V.S., Landl N.V. // IEEE Trans. Plasma Sci. 2012. Vol. 40. N 11. P. 2951-2960.

[31] Korolev Y.D., Frants O.B., Landl N.V., Kasyanov V.S., Galanov S.I., Sidorova O.I., Kim Y., Rosocha L.A., Matveev I.B. // IEEE Trans. Plasma Sci. 2012. Vol. 40. N 2. P. 535-542.

[32] Landl N.V., Korolev Y.D., Frants O.B., Geyman V.G., Bolotov A.V. // J. Phys. Conf. Series. 2015. Vol. 652. P. 012049. 